\title{
DIAMETER CALCULATION IN CONTACTLESS THREE-AXIS MEASURING DEVICES
}

\author{
Evgeny Fedorov", Alexander Koba \\ Tomsk Polytechnic University, Institute of Non-Destructive Testing, 634050, Tomsk, Russia
}

\begin{abstract}
The paper presents a three-dimensional method of measuring diameter and ovality of cylindrical objects in diverging laser beam. Mathematical software method is developed. Virtual simulation of this method and experimental testing are made. The results can be used for design and manufacture of optical devices, which will be used for technological control of external diameter of cable, wire, trumpet and other products.
\end{abstract}

\section{Introduction}

Various geometric and electrical parameters are used to assess the quality of cable products. The rejects can be minimized through the improved technology to control the product major characteristics, namely capacitance per unit length [1,2], eccentricity [3], outer diameter [4], insulation integrity [5] and others parameters [6-13].

Noncontact gauges for measuring the diameter of circular long-length objects such as cables, ropes, pipes, etc., which are based on the shadow measuring method using the divergent luminous flux, exhibit a number of undeniable advantages since they do not use the elements of lens and mirror optics. In particular, the measurement accuracy obtained by these devices using a combination of diffraction methods and statistical data processing may be of micrometer fractions [14]. However, when measurement is performed using the divergent light flux, the shadow dimensions of the measured object change in case the object is displaced within the test zone. This requires the use of complex ratios to calculate the true diameter of the object. Two-coordinate measuring gauges provide approximate values of the ovality estimated on the basis of the difference between the diameters of the two measuring axes, which are directly dependent on the object orientation within the measuring field. In this case, particular positions of the object do not allow the ovality fixation. Figure 1 shows an example of this orientation for an object with an ellipsoidal cross-section. The cross-section images $D x$ and $D y$ in the multi-element receivers of measuring channels $X$ and $Y$ are equal and they cannot provide the ovality parameters.

The measurements performed in the three-axis laser systems with a divergent laser beam can partially solve the above-stated problems. Three-axis measuring gauges exhibit the following advantages when compared with those of two-axis devices: three synchronized measuring axes on the same plane; detection of any deviation from the

*Corresponding author: evgeny_fyodorov@list.ru 
circular cross-section regardless of the object orientation in the measured zone; determination of the circumference and area of the cross-section.

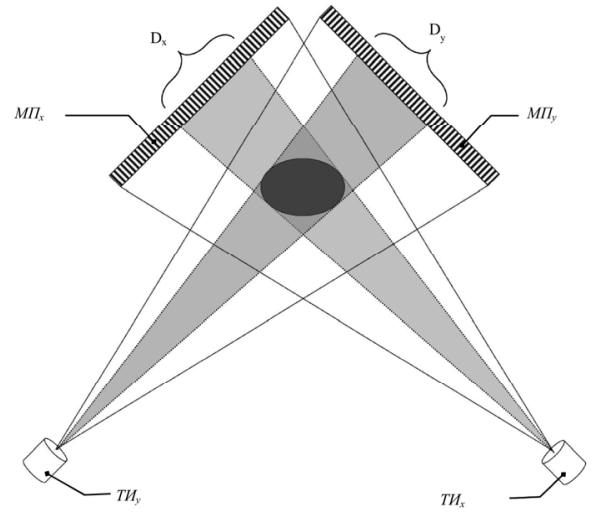

Figure 1. Example of orientation of the object with a non-circular cross-section, in which the calculation of ovality parameters is impossible. MRx, PEx and MRy, PEy are multi-element receivers and point emitters of measuring axes $\mathrm{Y}$ and $\mathrm{X}$, respectively. Dx and Dy are images of the object shadows in the corresponding photoreceivers.

\section{Diameter calculation}

Since the true diameter is a calculated value in both three- and two-axis laser systems, the derivation of mathematical expressions for its calculation is required.

Figure 2 shows the schematic diagram of the considered three-axis measuring gauge. The device comprises three measuring channels, with $X, Y$ and $Z$ axes arranged at $120^{\circ}$ angle to each other. Each channel has a point emitter (semiconductor laser) generating a divergent luminous flux and a multi-element photoreceiver measuring the size of the shadow falling on the object. The measuring object is found in the test zone; it is illuminated by three emitters and form three shadows in photoreceiver of the corresponding measuring channels.

The known design parameters of the device are as follows:

Distances $H_{x}, H_{y}$ and $H_{z}$ from the emitter center to the plane of the receiver of each channel: $\left(S_{X 0} ; B_{X 0}\right),\left(S_{Y 0} ; B_{Y 0}\right)$ and $\left(S_{Z 0} ; B_{Z 0}\right)$ segments.

Distances $H_{X 0}, H_{Y 0}$ and $\mathrm{H}_{Z 0}$ from the center of the emitter to the center of the test zone, i.e. the point $(0)$, which is an imaginary point for intersection of the measuring channel symmetry axes.

The step of the photoreceiver cells allows measurement of the shadow, i.e. its width, in the number of cells shadowed by the object, and the displacement of the shadow center relative to the "zero" cell of the receiver.

The known data are used to measure the object radii by the three channels $\left(R_{X}, R_{Y}, R_{Z}\right)$ and to determine the average radius $R_{a v}$ and the object ovality. To perform special measurements, data on the object position is required, (displacement of its $Z$ center) relative to the center of the gauge test zone (" 0 " point), i.e. the $E_{X}, E_{Y}$ and $E_{Z}$ values.

The problem is solved in three steps:

Step 1 - determination of the position of the $Z$ object center projection in the plane of the " $X$ ", " $Y$ " and " $Z$ " receivers.

The position of the center projection is characterized by the $X_{0}, Y_{0}$ and $Z_{0}$ values, which are $\left(B_{X 0} ; X_{0}\right),\left(B_{Y 0} ; Y_{0}\right)$ and $\left(B_{Z 0} ; Y_{0}\right)$ segments. According to the triangle $\left(S_{X 0} ; B_{X 0} ; X_{0}\right)$, it is known that: 


$$
\operatorname{tg}\left(\alpha+\alpha_{1}\right)=\frac{X_{0}}{H_{X}} \text {. Hence: } X_{0}=H_{X} \cdot \operatorname{tg}\left(\alpha+\alpha_{1}\right)
$$

The value $\alpha 1$ in expression (1) can be found through the triangle $\left(S_{X 0} ; B_{X 0} ; X_{I}\right)$ :

$$
\operatorname{tg} \alpha_{1}=\frac{X_{1}}{H_{Y}} ; \alpha_{1}=\operatorname{arctg} \frac{X_{1}}{H_{X}} .
$$

The angle $\alpha$ is determined through the triangle $\left(S_{X 0} ; B_{X 0} ; X_{2}\right)$ :

$$
\operatorname{tg}\left(\alpha+\alpha+\alpha_{1}\right)=\frac{X_{2}}{H_{X}} ;\left(2 \alpha+\alpha_{1}\right)=\operatorname{arctg} \frac{X_{2}}{H_{X}} .
$$

With regard to expression (2), the ratio of the angle takes the form:

$$
\alpha=\frac{1}{2}\left(\operatorname{arctg} \frac{X_{2}}{H_{X}}-\operatorname{arctg} \frac{X_{1}}{H_{X}}\right)
$$

Substitution of (3) and (2) in (1) and transformations result in:

$$
X_{0}=H_{X} \cdot \operatorname{tg} \frac{1}{2}\left(\operatorname{arctg} \frac{X_{2}}{H_{X}}+\operatorname{arctg} \frac{X_{1}}{H_{X}}\right)
$$

The same procedure is performed for $Y_{0}$ and $Z_{0}$ values:

$$
\begin{aligned}
& Y_{0}=H_{Y} \cdot \operatorname{tg} \frac{1}{2}\left(\operatorname{arctg} \frac{Y_{2}}{H_{Y}}+\operatorname{arctg} \frac{Y_{1}}{H_{Y}}\right) \\
& Z_{0}=H_{Z} \cdot \operatorname{tg} \frac{1}{2}\left(\operatorname{arctg} \frac{Z_{2}}{H_{Z}}+\operatorname{arctg} \frac{Z_{1}}{H_{Z}}\right)
\end{aligned}
$$

Similarity of the triangles $\left(S_{X 0} ; B_{X 0} ; X_{0}\right)$ and $\left(S_{X 0} ; O_{X} ; Z\right)$ provides:

Step 2 - determination of the $Z$ object center displacement relative to the center of the test zone. The displacement is characterized by $E_{x}, E_{y}$ and $E_{z}$ values (Figure 2).

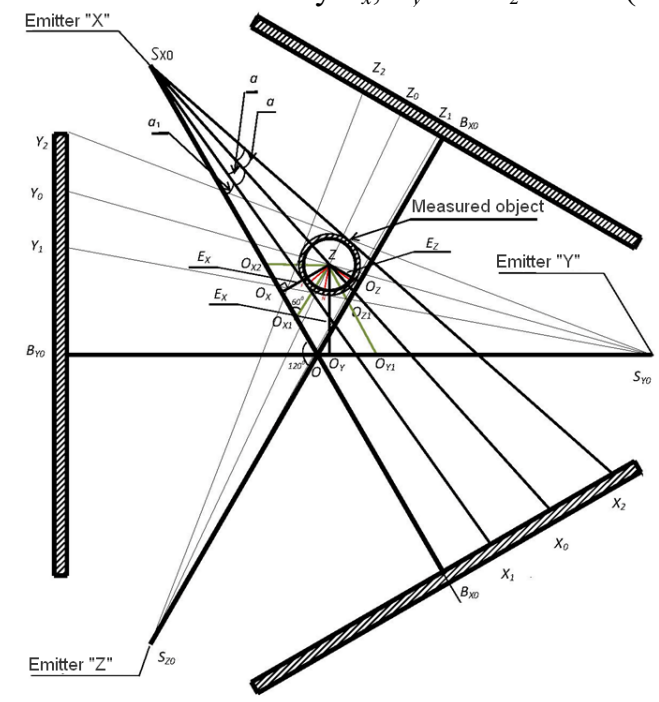

Figure 2. Calculation model for the device with three measuring axes.

$$
\frac{E_{X}}{X_{0}}=\frac{\left(S_{X 0} ; 0_{X}\right)}{H_{X}} \text {. Similarly: } \frac{E_{Y}}{Y_{0}}=\frac{\left(S_{Y 0} ; 0_{Y}\right)}{H_{Y}} ; \frac{E_{Z}}{Z_{0}}=\frac{\left(S_{Z 0} ; 0_{Z}\right)}{H_{Z}}
$$


Find the values for the segments $\left(S_{X 0} ; O_{X}\right),\left(S_{Y 0} ; O_{Y}\right)$ and $\left(S_{Z 0} ; O_{Z}\right)$ in (7), provided the segment $\left(0 ; 0_{X}\right)$ is:

$$
\begin{gathered}
\left(0 ; O_{X}\right)=\left(Z ; O_{Y 1}\right)-\left(O_{X 2} ; O_{X}\right)=\frac{E_{Y}}{\cos \left(\frac{\pi}{6}\right)}-\frac{E_{X}}{\operatorname{tg}\left(\frac{\pi}{3}\right)} ; \\
\text { segment }\left(0 ; O_{Y}\right)=\left(Z ; O_{X 2}\right)-\left(O_{Y} ; O_{Y I}\right)=\frac{E_{X}}{\cos \left(\frac{\pi}{6}\right)}-\frac{E_{Y}}{\operatorname{tg}\left(\frac{\pi}{3}\right)} . \\
\text { segment }\left(0 ; O_{Z}\right)=\left(Z ; O_{X I}\right)+\left(O_{Z} ; O_{Z 1}\right)=\frac{E_{X}}{\cos \left(\frac{\pi}{6}\right)}+\frac{E_{Z}}{\operatorname{tg}\left(\frac{\pi}{3}\right)} .
\end{gathered}
$$

Then:

$$
\begin{gathered}
\left(S_{X 0} ; O_{X}\right)=H_{X 0}-\frac{E_{Y}}{\cos \left(\frac{\pi}{6}\right)}+\frac{E_{X}}{\operatorname{tg}\left(\frac{\pi}{3}\right)} ;\left(S_{Y 0} ; O_{Y}\right)=H_{Y 0}-\frac{E_{X}}{\cos \left(\frac{\pi}{6}\right)}+\frac{E_{Y}}{\operatorname{tg}\left(\frac{\pi}{3}\right)} ; \\
\left(S_{Z 0} ; 0_{Z}\right)=H_{Z 0}+\frac{E_{X}}{\cos \left(\frac{\pi}{6}\right)}+\frac{E_{Z}}{\operatorname{tg}\left(\frac{\pi}{3}\right)}
\end{gathered}
$$

With regard to expression (8), ratios (7) take the form:

$$
\begin{gathered}
\frac{E_{X}}{X_{0}}=\frac{H_{X 0}-\frac{E_{Y}}{\cos \left(\frac{\pi}{6}\right)}+\frac{E_{X}}{\operatorname{tg}\left(\frac{\pi}{3}\right)}}{H_{X}} ; \frac{E_{Y}}{Y_{0}}=\frac{H_{Y 0}-\frac{E_{X}}{\cos \left(\frac{\pi}{6}\right)}+\frac{E_{Y}}{\operatorname{tg}\left(\frac{\pi}{3}\right)}}{H_{Y}} ; \\
\frac{E_{Z}}{Z_{0}}=\frac{H_{Z 0}+\frac{E_{X}}{\cos \left(\frac{\pi}{6}\right)}+\frac{E_{Z}}{\operatorname{tg}\left(\frac{\pi}{3}\right)}}{H_{Z}}
\end{gathered}
$$

Expressions (9) are a system of three equations with three unknowns: $E_{X}, E_{Y}$ and $E_{Z}$. Solve the equations and derive the expressions to calculate $E_{X}, E_{Y}$ and $E_{Z}$, in which the $X_{0}$, $Y_{0}$ and $Z_{0}$ values are calculated by (4), (5) and (6) ratios. This paper does not provide the extended expressions for calculation of the system of equations in letter values.

Step 3 - calculation of the object radii $R_{X}, R_{Y}$ and $R_{Z}$.

The schematic diagram in Figure 1 indicates that the $R_{X}$ radius, i.e. the segment $(F ; Z)$, is the side of the right triangle $\left(S_{X 0} ; Z ; F\right)$. According to this triangle:

$$
R_{X}=\left(S_{X 0} ; Z\right) \cdot \sin \alpha
$$

The other right triangle $\left(S_{X 0} ; Z ; O_{X}\right)$ is used to find the segment $\left(S_{X 0} ; Z\right)$ :

$$
\left(\mathrm{S}_{\mathrm{X} 0}, \mathrm{Z}\right)=\sqrt{\left(0_{X} ; Z\right)^{2}+\left(0_{X} ; S_{X 0}\right)^{2}}=\sqrt{E_{X}^{2}+\left(H_{X 0}-\frac{E_{y}}{\cos \left(\frac{\pi}{6}\right)}+\frac{E_{x}}{\operatorname{tg}\left(\frac{\pi}{3}\right)}\right)^{2}} .
$$

Substitute the value of the segment $\left(S_{X 0} ; Z\right)$ in (10) and use expression (3) for the angle to find the radius $R_{X}$. 


$$
R_{X}=\sqrt{E_{X}^{2}+\left(H_{X 0}-\frac{E_{y}}{\cos \left(\frac{\pi}{6}\right)}+\frac{E_{x}}{\operatorname{tg}\left(\frac{\pi}{3}\right)}\right)^{2}} \cdot \sin \frac{1}{2}\left(\operatorname{arctg} \frac{X_{2}}{H_{X}}-\operatorname{arctg} \frac{X_{1}}{H_{X}}\right)
$$

Similarly:

$$
\begin{gathered}
R_{Y}=\sqrt{E_{Y}^{2}+\left(H_{Y 0}-\frac{E_{X}}{\cos \left(\frac{\pi}{6}\right)}+\frac{E_{Y}}{\operatorname{tg}\left(\frac{\pi}{3}\right)}\right)^{2}} \cdot \sin \frac{1}{2}\left(\operatorname{arctg} \frac{Y_{2}}{H_{Y}}-\operatorname{arctg} \frac{Y_{1}}{H_{Y}}\right) \\
R_{Z}=\sqrt{E_{Z}^{2}+\left(H_{Z 0}+\frac{E_{X}}{\cos \left(\frac{\pi}{6}\right)}+\frac{E_{Z}}{\operatorname{tg}\left(\frac{\pi}{3}\right)}\right)^{2}} \cdot \sin \frac{1}{2}\left(\operatorname{arctg} \frac{Z_{2}}{H_{Z}}-\operatorname{arctg} \frac{Z_{1}}{H_{Z}}\right)
\end{gathered}
$$

The values of the $R_{X}$ and $R_{Y}$ radii being known, the average radius $R a v$ of the object and object ovality $(O)$ can be found.

$$
R_{a v}=\frac{\left|R_{X}\right|+\left|R_{Y}\right|+\left|R_{Z}\right|}{3} ; O=\left(R_{\max }-R_{\min }\right)
$$

\section{Method of evaluation}

A three-axis optical device for measuring the diameter of cylindrical objects has been simulated to verify the above suggested expressions (11-13). A computer model presented in Figure 2 comprises three identical measuring channels arranged at $120^{\circ}$ to each other. The initial geometric parameters to simplify calculations are the same for all the measuring channels:

- The distance from the emitter center to the plane of the receiver $H_{x}=H_{y}=H_{z}=300$ (mm).

- The distance from the emitter center to the test zone, i.e. the center of intersection of the measuring channel axes $H_{X 0}=H_{Y 0}=H_{Z 0}=220(\mathrm{~mm})$.

Such values of the optical devices geometric parameters are typical for the measurement area sufficient for most applications. In this model, the length of the working surface of multiple-receiver does not exceed $100 \mathrm{~mm}$. All the initial geometrical parameters and virtual positions of shadows in the radiation receiver planes in the corresponding channels $X_{1}, X_{2}, Y_{1}, Y_{2}, Z_{1}$ and $Z_{2}$, were determined in the computer model with $1 \mu \mathrm{m}$ error. Round objects with different diameters and different location in the virtual measuring zone were simulated to perform testing. When substituting the values obtained during the simulation into expressions (11-13), the measuring object diameters were calculated in the corresponding measuring axes $X, Y$ and $Z$. These calculated diameters $D_{x}, D_{y}, D_{z}$ for three arbitrary circular objects with the diameter $D$ are presented in Table 1 .

Table 1. Diameter values and the error for three axes.

\begin{tabular}{|c|c|c|c|c|c|c|c|}
\hline $\begin{array}{c}\text { Measuring } \\
\text { diameter } \boldsymbol{D} \\
(\mathbf{m m})\end{array}$ & $\begin{array}{c}\text { Diameter } \\
\boldsymbol{D}_{\boldsymbol{x}}(\mathbf{m m})\end{array}$ & $\begin{array}{c}\text { Error } \\
\boldsymbol{\Delta} \boldsymbol{D}_{\boldsymbol{x}} \\
(\boldsymbol{\mu m})\end{array}$ & $\begin{array}{c}\text { Diameter } \\
\boldsymbol{D}_{\boldsymbol{y}}(\mathbf{m m})\end{array}$ & $\begin{array}{c}\text { Error } \\
\boldsymbol{\Delta}_{\boldsymbol{x}} \\
(\boldsymbol{\mu \mathbf { m } )}\end{array}$ & $\begin{array}{c}\text { Diameter } \\
\boldsymbol{D}_{\boldsymbol{z}}(\mathbf{m m})\end{array}$ & $\begin{array}{c}\text { Error } \\
\boldsymbol{\Delta} \boldsymbol{D}_{\boldsymbol{x}} \\
(\boldsymbol{\mu \mathbf { m }})\end{array}$ & $\begin{array}{c}\text { Error } \\
\boldsymbol{\Delta} \boldsymbol{D}_{\boldsymbol{a} \boldsymbol{v}} \\
(\boldsymbol{\mu \mathbf { m } )}\end{array}$ \\
\hline 10.000 & 10.003 & -3 & 10.002 & -2 & 9.998 & 2 & -1 \\
\hline 17.000 & 16.997 & 3 & 16.999 & 1 & 17.002 & -2 & 0.67 \\
\hline 25.000 & 24.999 & 1 & 25.001 & -1 & 25.000 & 0 & 0 \\
\hline
\end{tabular}

The absolute error of the diameter calculation in the measuring axes $\Delta D_{x}, \Delta D_{x}$ and $\Delta D_{x}$, as well as the mean error $\Delta D_{a v}$ do not exceed $3 \mu \mathrm{m}$, that is acceptable with regard to the error in the initial parameter determination. In practice, the diameter calculation accuracy strongly depends on the accuracy of initial parameters. Further simulation of the three-axis 
device is to be performed on a real prototype using the methods of digital signal processing. Due to this, the error in the diameter calculation may be of micrometer fractions.

Further testing of the method and the proposed mathematical model of measurement was carried out using a real prototype of the diverging-laser-beam measuring device. The primary transducer of the diameter measuring gauge LDM-20 described in [13] was used to simplify the experiment. The test installation appearance is presented in Figure 3. The prototype is a system with a single optical measuring channel comprising the CCD (chargecoupled device) and the laser emitter. The transducer was mounted on the turntable capable of rotating by $360^{\circ}$ with a rotation angle accuracy of up to 1 arc minute. The object with the known diameter (calibre) was placed stationary in the center of the turntable. By rotating the table at the angles of $0^{\circ},-120^{\circ}$, and $+120^{\circ}$, a three-axis transducer was experimentally simulated.

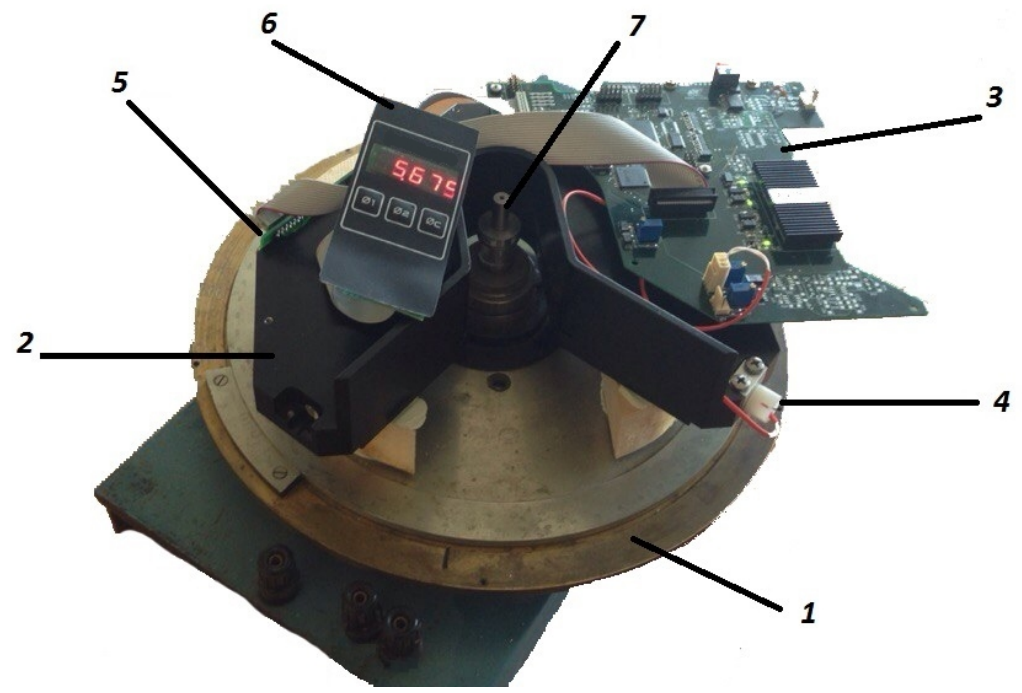

Figure 3. Test installation comprises: 1 - turntable, 2 - transducer housing, 3 - electronic control board, 4 - point emitter (semiconductor laser), 5 - CCD receiver board, 6 - display with control keys, 7 - object with the known diameter (caliber).

The geometrical parameters of the optical transducer were as follows:

- Distances from the emitter center to the plane of the receiver $H_{x}=H_{y}=H_{\mathrm{z}}=207.4$ $(\mathrm{mm})$.

- Distances from the emitter center to the center of the test zone $H_{X 0}=H_{Y 0}=H_{Z 0}=134$ (mm).

- The pixel size of the used CCD (NECuPD8871) was equal to $4 \mu \mathrm{m}$. The number of pixels was 10680 .

A simplified structure of the installation electronic part is illustrated in Figure 4. The linear CCD array and the point emitter (semiconductor laser) are controlled by the field programmable gate array (FPGA), which generates all necessary signal sequences. Analog data obtained from the CCD receiver arrive at the ADC. The data in the digital form are then accepted by the programmable array for conversion into the rising and falling edges of the shadow, which characterizes the linear dimensions of the measuring object. After this, the measured data are transmitted to the microcontroller via a conventional SPI interface. 


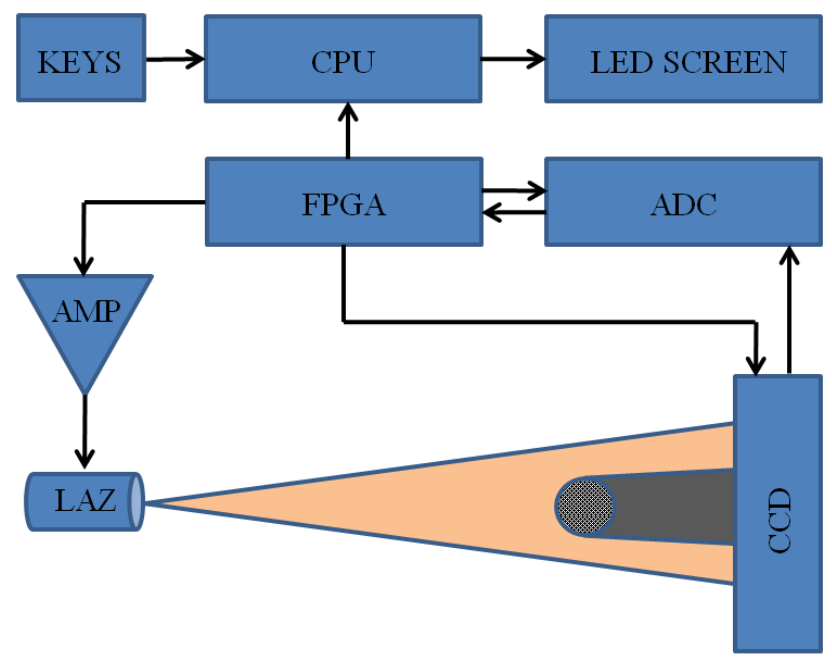

Figure 4. Block diagram of the test installation electronic part.

The microcontroller displays the data on a seven-segment LED indicator when you press the appropriate control keys on the keyboard. Due to this, positions of the shadow boundaries $\mathrm{X}_{1}, \mathrm{X}_{2}, \mathrm{Y}_{1}, \mathrm{Y}_{2}, \mathrm{Z}_{1}$ and $\mathrm{Z}_{2}$ were experimentally calculated. Similar to the computer model, the calculated values for three calibres with the known diameters were obtained using expressions (11-13). The results of the diameter calculation and calculation errors are given in Table 2.

Table 1. Diameters and errors obtained from the experimental data.

\begin{tabular}{|c|c|c|c|c|c|c|c|}
\hline $\begin{array}{c}\text { Caliber } \\
\text { diameter } \\
(\mathbf{m m})\end{array}$ & $\begin{array}{c}\text { Diameter } \boldsymbol{D}_{\boldsymbol{x}} \\
\mathbf{( \mathbf { m m } )}\end{array}$ & $\begin{array}{c}\text { Error } \\
\boldsymbol{\Delta} \boldsymbol{D}_{\boldsymbol{x}} \\
(\boldsymbol{\mu \mathbf { m } )}\end{array}$ & $\begin{array}{c}\text { Diameter } \boldsymbol{D}_{\boldsymbol{y}} \\
\mathbf{( \mathbf { m m } )}\end{array}$ & $\begin{array}{c}\text { Error } \\
\boldsymbol{\Delta} \boldsymbol{D}_{\boldsymbol{y}}(\boldsymbol{\mu m})\end{array}$ & $\begin{array}{c}\text { Diameter } \boldsymbol{D}_{z} \\
(\mathbf{m m})\end{array}$ & $\begin{array}{c}\text { Error } \\
\Delta \boldsymbol{D}_{z}(\boldsymbol{\mu \mathbf { m }})\end{array}$ & $\begin{array}{c}\text { Error } \\
\boldsymbol{\Delta} \boldsymbol{D}_{\boldsymbol{a} \boldsymbol{v}}(\boldsymbol{\mu \mathbf { m } )}\end{array}$ \\
\hline 14.842 & 14.832 & -10 & 14.839 & -3 & 14.847 & 5 & -2.7 \\
\hline 10.178 & 10.171 & -7 & 10.170 & -8 & 10.171 & -7 & -7.3 \\
\hline 12.900 & 12.905 & 5 & 12.901 & 1 & 12.891 & -9 & -1 \\
\hline
\end{tabular}

The absolute error of the diameter calculation in the measuring axes $\Delta D_{x}, \Delta D_{y}$ and $\Delta D_{z}$, as well as the mean error $\Delta D_{a v}$ insignificantly exceed the values obtained during simulation (Table 2), but remain within the range of $10 \mu \mathrm{m}$. This result is found to be in good agreement with simulation results and proves the validity of expressions (11-13) to calculate the diameter in tree-axis systems. Increased errors of the calculated diameter values obtained from the experimental data can be explained by the installation inaccuracy (Figure 3). Design of the three-axis measuring transducer resistant to deformation and use of the digital processing algorithms will enable the solution of the above-stated problems.

\section{Conclusion}

The paper proposes a method for three-axis measurement of the diameter using a diverging laser beam. Expressions (9, 11-14) have been derived to calculate the precise value of the object radii, the coordinates of the object center position, and the average radius and the precise value of its ovality in the maximum and minimum values. A computer modeling of the three-axis transducer, which data are shown to be in good agreement with experimental data, confirms the validity of the proposed conversion function. The described optical 
method and mathematical support can be used to design a primary measuring transducer for the device to test the diameter and ovality of long-length products.

\section{References}

[1] N.S. Starikova, V.V. Redko, G.V. Vavilova, J. Phys.: Conf. Ser. 671, 012056 (2016) doi: 10.1088/1742-6596/671/1/012056

[2] A.E. Goldshtein, G.V. Vavilova, V.Yu. Belyankov, Russ. J. Nondestr. Test. 51, 86 (2015) doi: 10.1134/S1061830915020047

[3] A.E. Goldshtein, E.M. Fedorov, Rus. J. Nondestr. Test. 46, 424 (2010) doi: $10.1134 / \mathrm{S} 1061830910060069$

[4] E.M. Fedorov, I.D. Bortnikov, Tech. Phys. 60, 1689 (2015) doi: $10.1134 / \mathrm{S} 1063784215110110$

[5] V.V. Red'ko, A.P. Leonov, L.A. Red'ko, V.A. Bolgova, J. Phys: Conf. Ser. 671, 012049 (2015) doi: 10.1088/1742-6596/671/1/012049

[6] E. Caetano, Á. Cunha, MATEC Web of Conferences 24, 01002 (2015) doi: $10.1051 / \mathrm{matec}$ onf $/ 20152401002$

[7] A.Y. Petrova, O.N. Chaikovskaya, I.V. Plotnikova, Tech. Phys. J. 60, 592 (2015) doi: $10.1134 / \mathrm{S} 1063784215040222$

[8] A.P. Surzhikov, T.S. Frangulyan, S.A. Ghyngazov, E.N. Lysenko, Journal of thermal analysis and calorimetry 102, 883 (2010) doi: 10.1007/s10973-010-0912-8

[9] O.V. Galtseva, S.V. Bordunov, N.M. Natalinova, S.V. Mazikov, IOP Conf. Ser.: Mater. Sci. Eng. 132, 012003 (2016) doi: 10.1088/1757-899X/132/1/012003

[10] A.M. Pritulov, R.U. Usmanov, O.V. Gal'Tseva, A.A. Kondratyuk, V.V. Bezuglov, V.I. Serbin, Russ Phys J+ 50, 187 (2007) doi: 10.1007/s11182-007-0026-3

[11] V.Y. Kazakov, D.K. Avdeeva, M.G. Grigoriev, N.M. Natalinova, I.V. Maksimov, M.V. Balahonova, BLM 7, 1 (2015)

[12] R. Kodermyatov, M. Ivanov, M. Yuzhakov, V. Kuznetsov, M. Yuzhakova, E. Timofeeva, MATEC Web of Conferences 48, 05004 (2016) doi: 10.1051/confmatec/20164805004

[13] A.A. Bespalko, A.P. Surzhikov, L.V. Yavorovich, P.I. Fedotov, Russ. J. Nondestr. Test. 48, 221 (2012) doi: 10.1134/S1061830912040043

[14] Y.A. Chursin, E.M. Fedorov, Optics\&Laser Technology 67, 86 (2015) doi: 10.1016/j.optlastec.2014.09.017 